INRA Prod. Anim., 2017, 30 (2), 93-106

\section{État des lieux et évolutions de la production bovine allaitante en France et dans trois pays européens}

\author{
M. LHERM ${ }^{1,3}$, J. AGABRIEL $L^{1,3}, J . D E V U N^{2,3}$
}

${ }^{\prime}$ UMR1213 Herbivores, INRA, Vetagro Sup, 63122, Saint-Genès-Champanelle, France 2 Institut de l'Élevage, Service Production de Viande, 63170, Aubière, France ${ }_{3}^{3}$ UMT SAFE Systèmes Allaitants Fourrages Environnement, 63122, Saint-Genès-Champanelle, France Courriel : michel.lherm@inra.fr

Actuellement, 34\% des vaches de l'Union Européenne (UE) à 28 sont conduites en système «bovin viande ». Ces systèmes sont présents principalement dans quatre pays (France, Espagne, Royaume-Uni, Irlande) qui regroupent $71 \%$ des vaches allaitantes de 1'UE. Présents notamment dans les zones défavorisées où ils participent fortement au développement rural, ils se sont étendus après la mise en place des quotas laitiers et l'instauration de primes spécifiques couplées. Cet article illustre les stratégies variées des pays pourtant soumis globalement à la même politique agricole commune.

Le développement en Europe et en France de la production bovine allaitante spécialisée a commencé au début des années 1960. Auparavant, les bovins étaient élevés pour la production laitière et dégageaient un coproduit viande important, essentiellement sous forme de veaux de boucherie et de vaches de réforme. Les races à viande, comme la Charolaise, étaient également utilisées pour la traction animale et la production de bœufs d'embouche. Mais suite au développement et à la généralisation de la mécanisation et l'industrialisation des filières au sortir de la deuxième guerre mondiale, ces systèmes d'élevage ont subi de profonds changement et se sont spécialisés (cf. Cerles et al ce numéro). Les spécialisations laitière et allaitante des systèmes de production se sont alors accélérées notamment suite à la mise en place des quotas laitiers européens en 1984 et à l'instauration de la Prime au Maintien des Troupeaux de Vaches Allaitantes (PMTVA) en 1980 en France.

En parallèle les territoires d'Europe et de France se sont aussi globalement spécialisés. L'arc atlantique et le grand ouest français sont devenus des zones où prédomine la production laitière intensive, alors que le Massif central en profitant de ses races spécialisées, s'est orienté majoritairement vers la produc- tion d'animaux mâles « maigres » pour l'Italie et l'Europe du sud où se sont développés des ateliers d'engraissement. L'agrandissement des exploitations, la mécanisation, la modification des pratiques ont permis de gagner en efficacité du travail au risque parfois de diminuer les performances techniques (Bébin et al 1995). Désormais dans un contexte économique, où le cadre réglementaire de la PAC, la démographie agricole et les attentes sociétales des éleveurs évoluent, les exploitations d'élevage bovin viande s'adaptent pour préserver leur revenu face au ciseau ${ }^{1}$ des prix (Lemery et al 2005, Dedieu et Ingrand 2010, Hocquette et Chatellier 2011), tout en s'ajustant au marché. Dans ce but, elles continuent de faire évoluer leurs dispositifs de production. Elles ont gagné en productivité du travail à la faveur de davantage d'équipements, bâtiments et cheptels. Ces investissements se sont souvent accompagnés d'endettement qui a limité le revenu disponible pour les prélèvements privés.

Aujourd'hui, le cheptel bovin viande européen, contribue pour environ $40 \%$ à la production européenne de viande bovine qui atteint 7,7 millions de tonnes équivalent carcasse en 2015 , et pour $11 \%$ à la production mondiale. Cependant, du fait de la baisse du nombre de Vaches
Laitières (VL) et de la production de viande qui lui est associée, l'UE est devenue déficitaire de l'ordre de $5 \%$ de sa consommation intérieure. En 2014, le solde commercial est négatif de 34000 tonnes équivalent-carcasse (tec) de viande bovine et de 629 millions d' $€$ (Dumont et al 2016). Les exportations européennes de viande bovine ont considérablement diminué au fil du temps, en passant de 1,2 million de tec en 1990 à seulement 200000 tec en 2013. Les importations sont, quant à elles, restées assez stables (Roguet et al 2015, Chatellier 2016). Par ailleurs, les habitudes de consommation évoluent en UE comme en France où on constate, selon FranceAgriMer, une baisse tendancielle de la consommation individuelle de viande bovine de $0,8 \%$ par an depuis 1980 . De plus, le type de produit de viande recherchée se modifie, l'augmentation de la demande porte essentiellement sur la viande hachée.

Cet article introductif vise à « planter le décor » de la production allaitante au niveau français et européen (Chatellier et al 2000, Sarzeaud et al 2008) en rappelant les principales caractéristiques du contexte concernant les moyens de production mis en œuvre (surfaces, cheptel, travail, capital), les systèmes fourragers (composition, chargement). Au niveau européen, nous nous focaliserons en plus

${ }^{1}$ La notion de ciseau recouvre une image qui traduit le fait que le prix des charges augmente plus vite que celui des produits. 
de la France sur les trois principaux pays concernés par cette production (Espagne, Royaume-Uni et Irlande) qui ont des structures et dynamiques différentes. Les performances et résultats économiques qui rémunèrent les facteurs de productions de l'exploitation (travail, capitaux propres) seront ensuite analysées au regard des intrants utilisés.

\section{1 / Les exploitations agricoles françaises avec des bovins à viande et leur diversité}

\section{1 / Un élevage présent sur la quasi-totalité du territoire au sein d'exploitations plus ou moins spécialisées}

L'élevage bovin viande est présent sur l'ensemble du territoire français, mais avec une densité très variable d'une région à l'autre. La majorité des exploitations est en effet concentrée géographiquement, notamment dans le grand Massif central souvent appelé « bassin allaitant ", les Pays de la Loire et le Nord des Deux-Sèvres, ainsi que dans les zones de piémont des Pyrénées. Ces bassins regroupent 50\% des 4100000 Vaches Allaitantes (VA) recensées en 2010. Le reste du cheptel allaitant se répartit entre les zones d'élevage de l'Ouest (12,5\% des vaches) et les zones herbagères du Nord-Ouest et du Nord-Est (12,5\% des vaches également). Le solde se rencontre dans les zones de polyculture-élevage des bassins parisien et aquitain, les zones à dominante céréalière et les zones de montagne.

Les troupeaux détenus par ces exploitations sont de taille très variable, (tableau 1). Globalement, ils contribuent à hauteur de deux tiers du tonnage de viande produit en France et à l'exportation d'environ 1 million de broutards mâles et femelles chaque année. Ce nombre a tendance à baisser depuis 5 ans du fait d'un tassement de la demande italienne en partie lié à la baisse de la consommation et à la concurrence d'autres pays d'Europe.
Selon les zones de production, les systèmes d'exploitation sont plus ou moins spécialisés, en lien notamment avec la gamme des possibles et des potentialités de cultures. Les données du recensement agricole (RA 2010) ont permis ainsi de classer l'ensemble des exploitations avec bovins en 6 grandes familles selon l'importance du Produit Brut Standard de chaque production (figure 1)

i) les exploitations spécialisées bovin viande pour lesquelles l'élevage bovin viande est l'activité principale (Herbivores) ,

ii) les exploitations « grandes cultures et élevage bovin viande en complément » (Grandes cultures dominantes);

iii) les exploitations de type polyculture et élevage bovin viande, où les deux activités sont d'importance comparable (Polyculture-élevage) ;

iv) les exploitations associant l'élevage bovin viande et des granivores en importance comparable (Mixtes granivores) v) les exploitations " granivores et élevage bovin en complément » (Dominantes granivores);

vi) les exploitations « cultures spéciales et/ ou pérennes et élevage bovin viande » (Cultures pérennes \& spéciales).

\section{2 / Des systèmes d'élevage divers}

L'élevage bovin viande est également caractérisé par une diversité de races (figure 2) avec chacune leurs propres caractéristiques. La race Charolaise avec 1,5 million de vaches est prédominante et représente $37 \%$ du cheptel national. Elle est principalement localisée en Bourgogne, dans le Nord du Massif central, ainsi que dans les Pays de la Loire. La race Limousine (1 million de vaches soit $26 \%$ ) est naturellement très représentée dans le Limousin et son pourtour ouest. La Blonde d'Aquitaine, troisième race en effectif $(0,5$ million de vaches soit $12 \%$ ) est essentiellement présente dans le Sud-Ouest, mais aussi maintenant dans l'ouest de la France. Enfin, les races rustiques (Salers, Aubrac, 0,2 millions chacune) sont très présentes

Figure 1. Répartition par département des exploitations avec bovins viande ayant plus de 20 de Vaches Allaitantes (VA) sans Vaches Laitières (VL) selon les combinaisons de productions (d'après données RA 2010, traitement Institut de l'Élevage, Agabriel et al 2014).

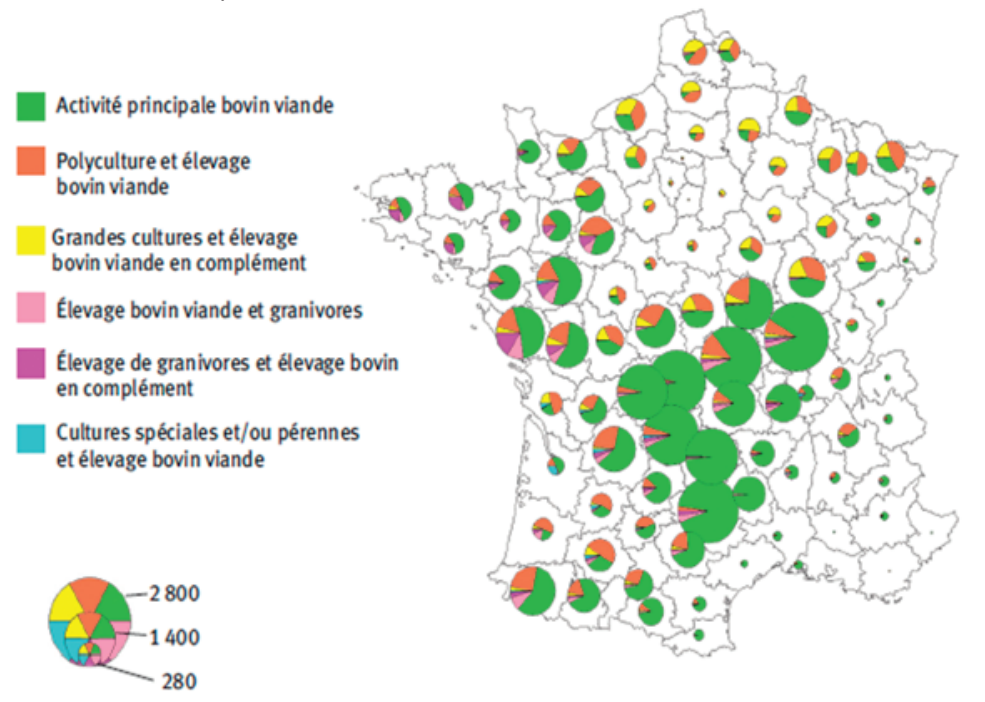

Tableau 1. Répartition des exploitations et des vaches allaitantes selon le type d'exploitation (Agabriel et al 2014).

\begin{tabular}{|l|c|c|c|c|c|}
\hline \multirow{2}{*}{ Type d'exploitation } & \multicolumn{2}{|c|}{$\begin{array}{c}\text { Exploitations ayant des } \\
\text { Vaches Allaitantes }\end{array}$} & \multicolumn{3}{|c|}{$\begin{array}{c}\text { Nombre } \\
\text { de Vaches Allaitantes }\end{array}$} \\
\cline { 2 - 6 } & Nombre & $\%$ & Moyenne & \multicolumn{1}{|c|}{ Total } & 78 \\
\hline Allaitants > = 20 VA & 56900 & 47 & 56,4 & 3206000 & 7 \\
\hline Allaitants < 20 VA & 25300 & 21 & 11,2 & 284000 & 11 \\
\hline Mixtes lait-viande & 19600 & 16 & 22,7 & 444000 & 3 \\
\hline Autres élevages (ovins, caprins...) & 9500 & 8 & 15,0 & 143000 & 1 \\
\hline Très petits élevages (<5 VA) & 9900 & 8 & 2,3 & 23000 & 1 \\
\hline TOTAL & $\mathbf{1 2 1 2 0 0}$ & $\mathbf{1 0 0}$ & $\mathbf{3 3 , 8}$ & $\mathbf{4 1 0 0 ~ 0 0 0}$ & $\mathbf{1 0 0}$ \\
\hline
\end{tabular}


Figure 2. Répartition des vaches allaitantes par département selon les races (d'après données RGA 2010, traitement Institut de l'Élevage).

(Source : BDNI - traitement Institut de l'Élevage).

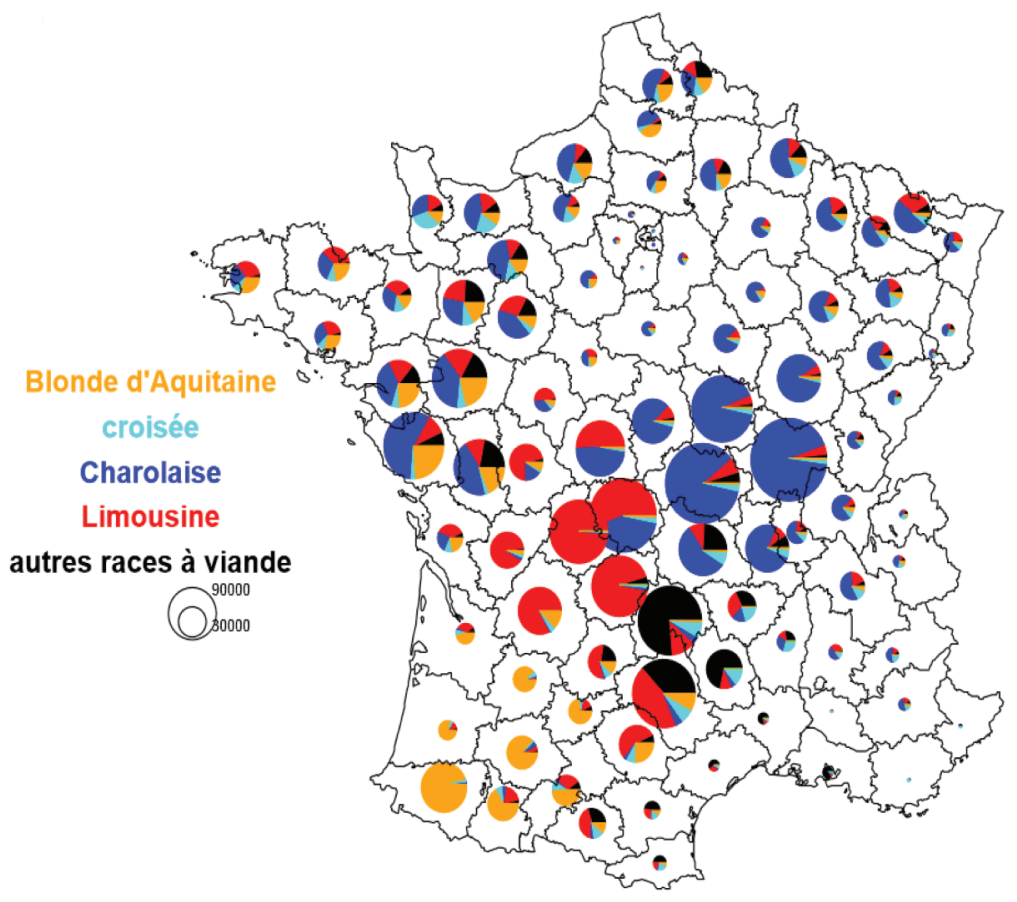

dans le Sud du Massif central et gagnent dans d'autres zones comme la Normandie. Ces types génétiques présentent des gabarits et des aptitudes laitières contrastées, qui influencent les besoins alimentaires et les densités énergétiques des régimes qui leurs sont nécessaires.

Au niveau français, qu'ils soient associés ou non à d'autres productions (figure 1), les grands types de systèmes d'élevage bovin viande, peuvent se distinguer, et se classer par les combinaisons des catégories de mâles et de femelles commercialisées :

i) les systèmes avec production de jeunes broutards et femelles maigres ou finies. Les mâles sont vendus au sevrage à des âges variant de 6 à 9 mois, selon les races et zones de production. Les génisses non conservées pour le renouvellement sont vendues maigres (soit au sevrage ou après une période de repousse pouvant aller jusqu'à un deuxième passage à l'herbe) ou finies pour la boucherie. Les vaches sont aussi vendues, soit maigres soit finies. Ces deux types regroupent près de $43 \%$ des exploitations bovin viande ayant plus de $20 \mathrm{VA}$ sans VL (RA 2010) ;

ii) les systèmes avec production de mâles maigres lourds et femelles maigres ou finies. Les mâles sont vendus après une période de repousse (broutards lourds ou repoussés) qui peut intégrer un deuxième passage à l'herbe (taurillons d'herbe dénommés « bourrets » dans l'Aubrac). Les génisses non conservées pour le renouvellement et les vaches de réforme sont également vendues maigres ou finies. Ces deux types regroupent près de $21 \%$ des exploitations ayant plus de $20 \mathrm{VA}$;

iii) les systèmes avec production de jeunes bovins engraissés, et dans la majorité des cas des femelles finies, regroupant de l'ordre de $17 \%$ des exploitations ayant plus de $20 \mathrm{VA}$. Les mâles sont engraissés majoritairement à l'auge, à partir du sevrage, et plus rarement après un deuxième passage à l'herbe. Les génisses peuvent être engraissées à l'herbe avec une complémentation ou à l'auge et abattues entre 24 et 38 mois selon les débouchés. Certaines, en particulier en Limousin, sont mises en finition dès le sevrage et abattues entre 12 et 20 mois ;

iv) les systèmes avec production de veaux sous la mère (veaux de boucherie mâles ou femelles, vendus entre 3 mois et 6 mois) et de veaux lourds (veaux mâles ou femelles vendus à la boucherie entre 6 mois et 10 mois), regroupant de l'ordre de $8 \%$ des exploitations ayant plus de $20 \mathrm{VA}$;

v) les systèmes avec production de boufs et dans la majorité des cas des femelles finies, ne regroupant plus que de l'ordre de $2 \%$ des exploitations ayant plus de 20 VA. Les génisses sont engrais- sées à l'herbe avec une complémentation ou à l'auge et abattues vers 30-36 mois ;

vi) les systèmes engraisseurs spécialisés regroupent les élevages sans cheptel reproducteur. Plus présent dans le Nordest et l'Ouest de la France, ils sont proches d'un millier et finissent essentiellement des jeunes bovins à l'auge à partir du sevrage ou courte repousse.

$\mathrm{Au}$ final seules $9 \%$ des exploitations de plus de 20 VA ne sont pas classées dans cette typologie car n'ayant pas de type bien défini et stable. Cette diversité des systèmes d'élevage de bovins allaitants français est le résultat d'un compromis entre la demande des marchés (catégories d'animaux), le dynamisme des filières au sein des bassins de production et les potentialités de production de ressources alimentaires. Les choix des éleveurs interviennent également dans la conception et la mise en œuvre du système fourrager associé qui dépend aussi du contexte socio-économique et des spécificités propres à chaque exploitation.

\section{3 / Un important accroissement de la taille et de la productivité du travail}

De 2,3 millions en 1970, l'effectif VA atteint 2,8 millions en 1979 à la veille de l'instauration de la PMTVA, puis 2,9 en 1983 avant la mise en place des quotas laitiers. Il atteint un sommet en 20002001 avec 4,2 millions d'individus. Depuis l'effectif de VA fluctue légèrement autour de 4 millions avec un minimum en 2004 et un maximum en 2008 quasiment retrouvé actuellement (figure 5).

Depuis 1988 (tableau 2), le nombre d'élevages allaitants s'est à peu près maintenu, les nouveaux arrivants dans cette production, en particulier ceux qui ont converti les VL en VA, compensant les cessations d'activité. Dans ces exploitations, la main-d'œuvre est restée stable : - 0,11 Unité de Travail Familial (UTF) et $+0,02$ salarié, pendant que les structures (surface, troupeau) grossissaient fortement pour dépasser 100 ha et approcher les 120 Unités Gros Bovin (UGB) avec $70 \mathrm{VA}$. On notera une grande stabilité dans le chargement (1,3 UGB/ha de Surface Fourragère Principale (SFP)) et la part de Surface Toujours en Herbe (STH) (51 à 53\% de la Surface Agricole Utile (SAU)). La longueur du cycle de production mesuré par le nombre d'UGB par VA s'est réduit, ce qui traduit l'abandon déjà engagé des produits bovins âgés comme les bœufs en charolais au profit de broutards destinés à la production de jeunes bovins. La productivité du travail mesurée par la taille de l'appareil de 
Tableau 2. Évolution entre 1988 et 2014 de quelques caractéristiques des élevages allaitants (OTEX 46, moyens et grands) français. (Source : RICA France).

\begin{tabular}{|l|r|r|c|}
\hline & $\mathbf{1 9 8 8}$ & $\mathbf{2 0 1 4}$ & \% évolution \\
\hline Nombre d'exploitations & 33149 & 32834 & -2 \\
\hline Nombre d'UTA & 1,46 & 1,37 & -6 \\
\hline \multicolumn{1}{|c|}{ dont salariés } & 0,07 & 0,09 & +30 \\
\hline SAU (ha) & 58,3 & 107,2 & +84 \\
\hline dont \% STH & 51,1 & 52,6 & +3 \\
\hline Nombre de VA & 33,2 & 67,8 & +104 \\
\hline Nombre d'UGB & 65,8 & 117,9 & +79 \\
\hline Chargement UGB/SFP & 1,3 & 1,3 & $=$ \\
\hline UGB/VA & 1,98 & 1,74 & -12 \\
\hline SAU/UTA & 40 & 78 & +96 \\
\hline UGB/UTA & 45 & 86 & +91 \\
\hline
\end{tabular}

UTA : Unité de Travail Annuel ; SAU : Surface Agricole Utile ;

STH : Surface Toujours en Herbe ; VA : Vaches Allaitantes ; UGB : Unité Gros Bovin ; SFP : Surface Fourragère Principale.

production ramené à la main-d'œuvre disponible - Unité de Travail Annuel (UTA) - (SAU/UTA et UGB/UTA) a quasi doublé, soit un accroissement moyen de 3,7 et 3,5\% par an. Cette forte augmentation ne s'est pourtant pas accompagnée de dégradation de la productivité par vêlage qui est restée à un niveau proche du potentiel biologique (de l'ordre de 95 veaux sevrés pour 100 vêlages pour les principales races à viande (Idele 2015)). Les risques de mortalité des jeunes animaux la première année de vie sont quasi stables et voisins de 10\% (Perrin et al 2011).

\section{2 / Contexte de production européen : principaux systè- mes de production et systèmes fourragers $^{2}$}

L'UE à 28 compte en 2013 près de 36 millions de vaches dont 12,3 millions, soit $34 \%$ qui sont conduites en système bovin viande (allaitant sans traite). À l'échelle européenne, le cheptel de vaches allaitantes est géographiquement plus concentré que le cheptel laitier. La France avec plus de 4 millions de vaches allaitantes dispose ainsi de 32\% de l'effectif de l'UE en 2013. Les pays d'Europe possédant un cheptel significatif de vaches allaitantes sont l'Espagne et le Royaume-Uni (RU) avec près de $15 \%$ de l'effectif européen chacun, puis l'Irlande avec $10 \%$. Au total ces 4 pays représentent $71 \%$ du cheptel allaitant de l'UE (figure 3).
En France les systèmes bovin viande se rencontrent principalement dans les zones herbagères souvent défavorisées où la part de prairies permanentes dans la SAU atteint les niveaux les plus élevés (Roguet et al 2015). En Espagne les trou-

peaux se situent dans les montagnes de Galice, du Pays Basque et dans les zones des plateaux secs d'Estramadure. C'est au niveau de la Corniche cantabrique que les densités sont les plus fortes. $\mathrm{Au}$ RU, le cheptel est assez diffus en dehors

Figure 3. Densité de vaches allaitantes dans l'UE-27 en 2010 exprimée en nombre de vaches par $\mathrm{km}^{2}$ à l'échelle des zones Nuts3.

(Source : Eurostat - Traitement RMT Économie des filières animales), (Roguet et al 2015).

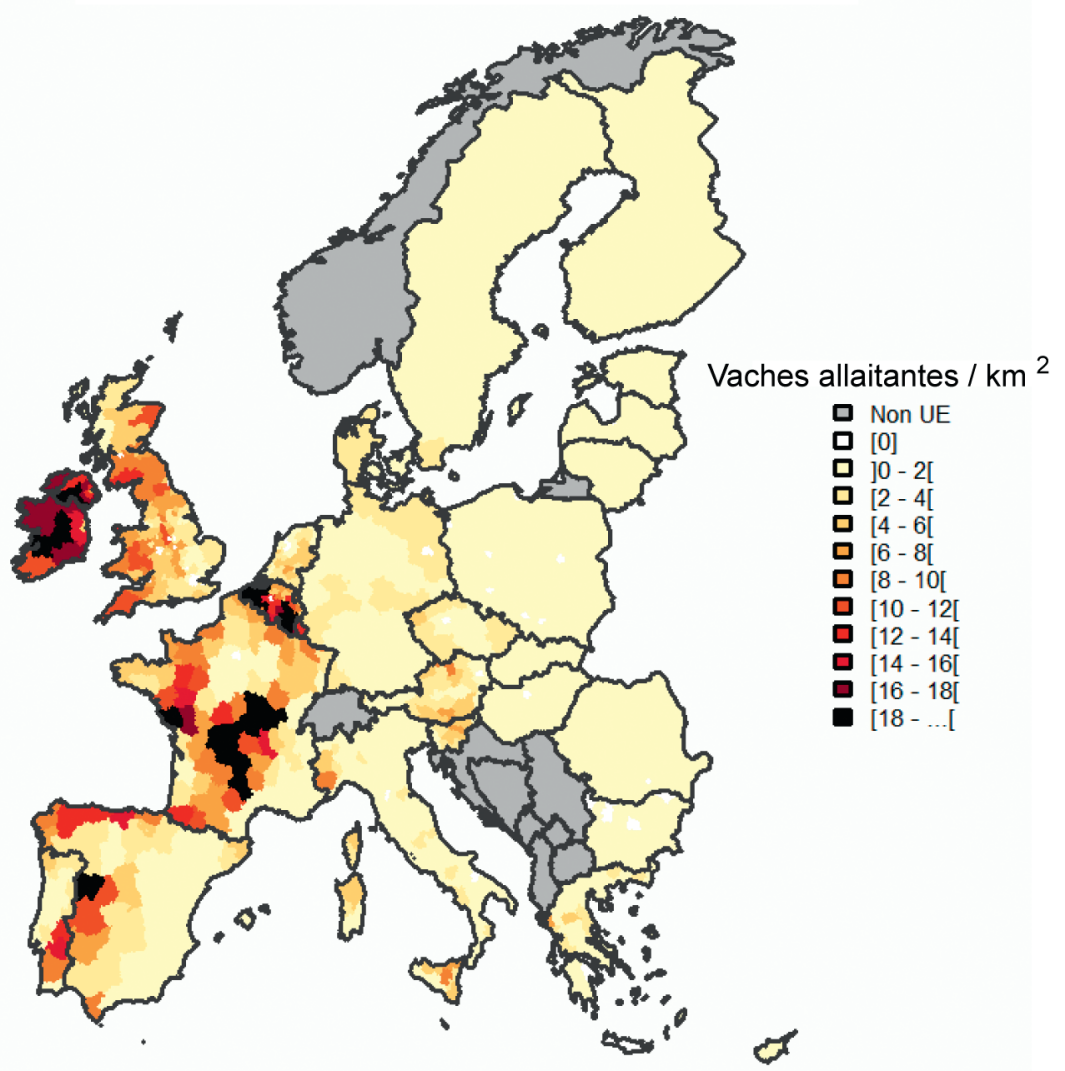

${ }^{2}$ Les données de ce chapitre proviennent de la base européenne : Eurostat http://ec.europa.eu/eurostat/web/agriculture/data/database 
du quart sud-est orienté vers les cultures. Les densités les plus élevées sont en Écosse, dans le Yorkshire, le Pays de Galles et en Cornouailles. Ils sont ainsi plutôt positionnés dans les zones extensives de collines. En Irlande, l'élevage allaitant est dense dans le centre du pays.

Les contextes pédoclimatiques sont tous assez différents par la période et la durée de croissance de l'herbe et les conditions climatiques hivernales (durée, température, neige) qui imposent ou non un hivernage en bâtiments. Les dates de mises bas en dépendent et sont majoritairement positionnées à l'automne en Europe du sud, et au contraire en hiver - début de printemps en lien avec la pousse de l'herbe dans le nord-ouest plus humide.

\section{1 / Les grandes évolutions des troupeaux et des effectifs dans les quatre principaux pays détenteurs de bovins allaitants}

Le nombre de vaches laitières a diminué dans tous les pays d'Europe et plus particulièrement à la suite de la mise en place des quotas laitiers en 1984. La spécialisation des exploitations, les investissements nécessaires (notamment les équipements de traite) et la forte augmentation de la production laitière par vache $(+100$ l/an) en sont les principaux moteurs. Par ailleurs, on peut également signaler la mise en place des soutiens spécifiques aux troupeaux de VA (PMT VA) initiés en France en 1980 puis généralisés aux autres pays de l'UE dans le cadre de la réforme de la Politique Agricole Commune (PAC) de 1992. En 1984, 1'UE à 11 comptait 27 millions de VL et 7 millions de VA (soit 34 millions de vaches), et en 201517 millions de VL $(-38 \%)$ et 10,5 de VA $(+50 \%)$ (soit au total 28 millions de vaches, - 18\%). Ainsi 3,5 millions de VA ont remplacé 10,4 millions de VL, soit un taux de remplacement d'un tiers seulement. Mais des nuances dans les dynamiques nationales sont à noter avec des conséquences contrastées selon les pays (figure 4). Ainsi, le « remplacement » partiel de vaches laitières par des vaches allaitantes a été plus ou moins prononcé selon les pays (figure 5) entre 1984 et 2015 :

i) au Royaume-Uni en raison des crises liées à l'encéphalite spongiforme bovine (ESB) la hausse des effectifs de VA est quasi-nulle $(+2 \%)$. Ce pays a aussi perdu 200000 VA depuis 2005 depuis le découplage total de la PMTVA;

ii) en France, le cheptel de VA en hausse depuis 1970, gagne 1 million de têtes $(+33 \%)$ passant de 3 à 4 millions, 3 VL sont ainsi remplacées par seulement 2 VA; iii) le développement du cheptel allaitant, est important en Espagne passant de 0,8 à 2 millions $(+147 \%)$ et en Irlande de 0,4 à 1 millions $(+151 \%)$ alors que le cheptel laitier Irlandais diminue peu. Ce pays a bénéficié fortement de son entrée en Europe et a profité de la hausse des importations britanniques suite au déclin de son cheptel pour accrô̂tre ses productions bovines;

iv) les autres pays ne développent pas leur troupeau allaitant comme la Pologne, ou peu comme l'Allemagne dont le cheptel atteint $600000 \mathrm{VA}$ pour 4 millions de VL. Dans ces pays les systèmes d'élevage bovin se sont moins spécialisés en raison de l'utilisation de races mixtes comme la Pie Rouge dont la viande représente un coproduit significatif. L'Allemagne consomme peu de viande bovine $(14 \mathrm{~kg} /$ habitant/an vs 24 en France), sa production issue du troupeau laitier suffit et permet même d'exporter vers la France, d'où une présence restreinte des troupeaux allaitants à ses zones très défavorisées qui se situent surtout dans les nouveaux «landers» du nord-est (Stolz 2014).

La répartition géographique de l'élevage allaitant européen (figure 3 ) est le fruit de deux dynamiques spatiales inverses qui se sont succédées depuis le milieu des années 1980. Lors de l'instauration des quotas laitiers en 1984, de nombreuses petites exploitations ont été incitées à arrêter la production laitière faute d'un quota suffisant. Une partie d'entre elles s'est alors reconvertie dans l'élevage allaitant. À partir des années 1990, la mise en place de la PMTVA est venue conforter cette évolution et, jusqu'en 2005 date à laquelle est intervenu son découplage dans certains pays, un certain nombre de petits producteurs laitiers européens dispersés géographiquement ont échangé leur quota contre des droits à primes PMTVA ou ont été amenés à développer un troupeau allaitant pour valoriser les surfaces, notamment en herbe, libérées par la baisse des effectifs laitiers (Dumont et al 2016). À partir des années 2000, l'élevage bovin allaitant a eu tendance à se recentrer progressivement dans ses bassins d'origine et dans les zones herbagères à fortes contraintes. Au RoyaumeUni et en Irlande, le découplage total de la PMTVA depuis 2003 a contribué à la décapitalisation du cheptel allaitant et à sa concentration dans les zones où la compétition avec les autres productions, notamment la production laitière, était la moins forte. A contrario, en France et en Espagne, le couplage (partiel) de la PMTVA participe à l'ancrage de la production bovine allaitante sur les territoires. Cette tendance à la relocalisation des vaches allaitantes dans les zones défavorisées a été confortée par les aides directes attribuées dans le cadre du second pilier de la PAC, notamment l'ICHN (Indemnité Compensatoire de Handicaps Naturels) et la PHAE (Prime Herbagère Agro-Environnementale) (Dumont et al 2016).

En France l'élevage allaitant s'oriente surtout vers la production de génisses et de jeunes bovins (ou broutards) destinés à être engraissés dans le pays ou exportés en maigre, notamment vers l'Italie. La production allaitante espagnole est essentiellement constituée de très jeunes broutard(e)s, envoyé(e)s vers des centres d'engraissement spécialisés pour leur finition sur le modèle des «feed-lots» américains. Dans les îles Britanniques, l'élevage allaitant produit principalement des bœufs de deux ans et des génisses, même si la production de jeunes bovins tend désormais à se développer.

Figure 4. Évolution entre 2005 et 2013 des effectifs de vaches nourrices en Europe (d'après Eurostat).

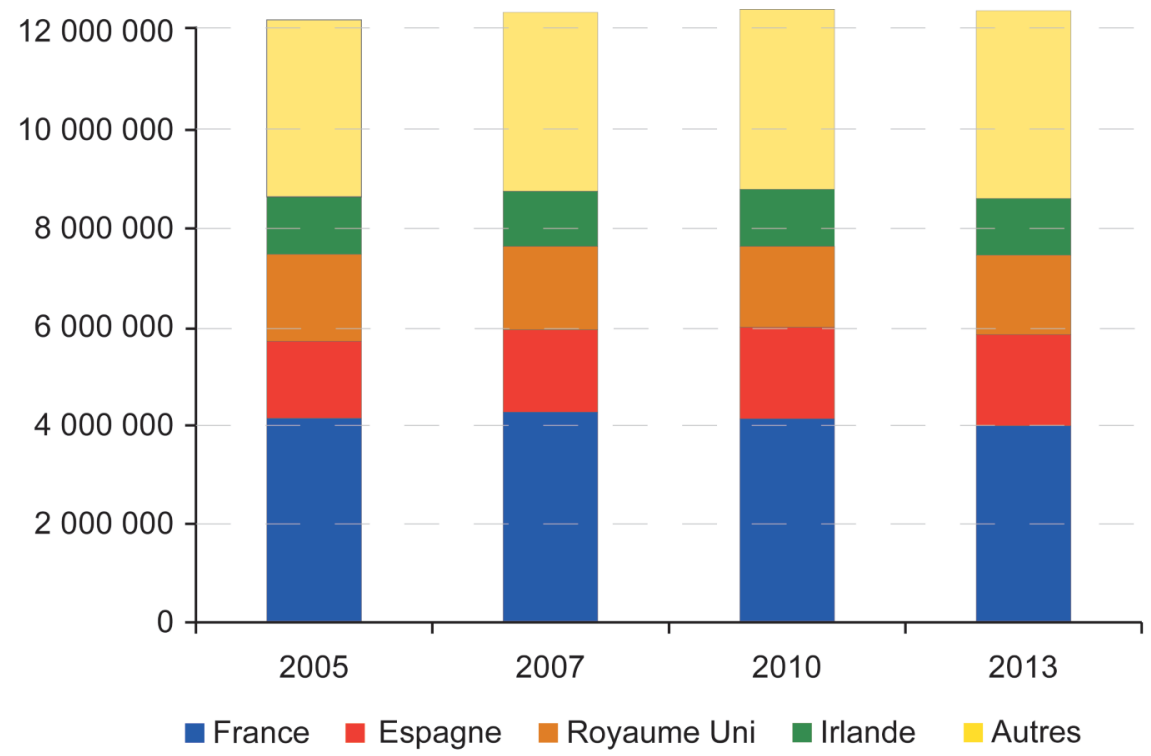


Figure 5. Évolution entre 1970 et 2016 des effectifs de vaches laitières (en bleu, le point jaune indique la mise en place des quotas laitiers) et allaitantes (en rouge) dans 6 pays de l'UE (d'après Eurostat).

France

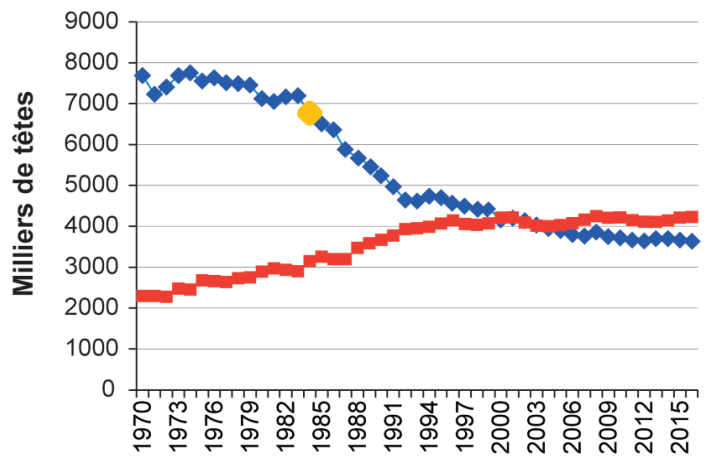

Espagne

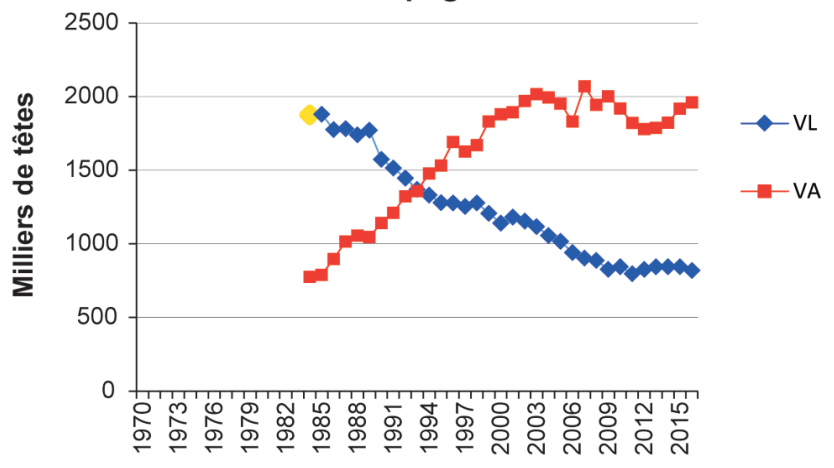

Allemagne (RFA jusqu'en 1990)

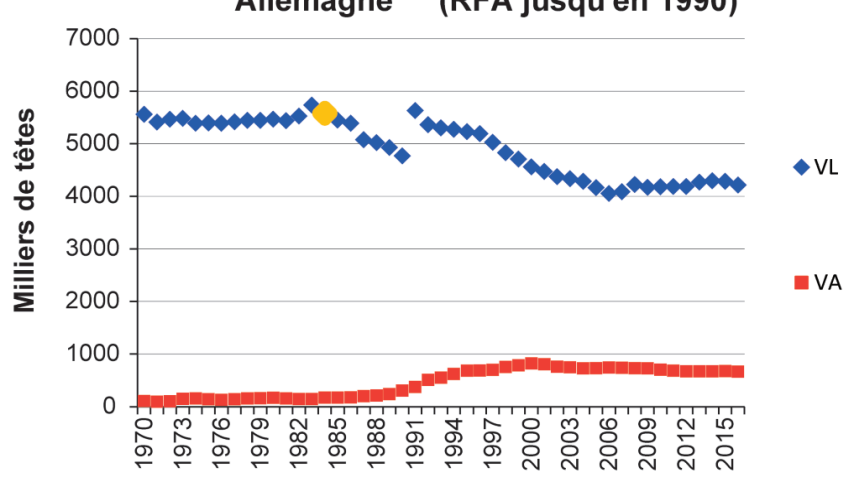

Royaume-Uni
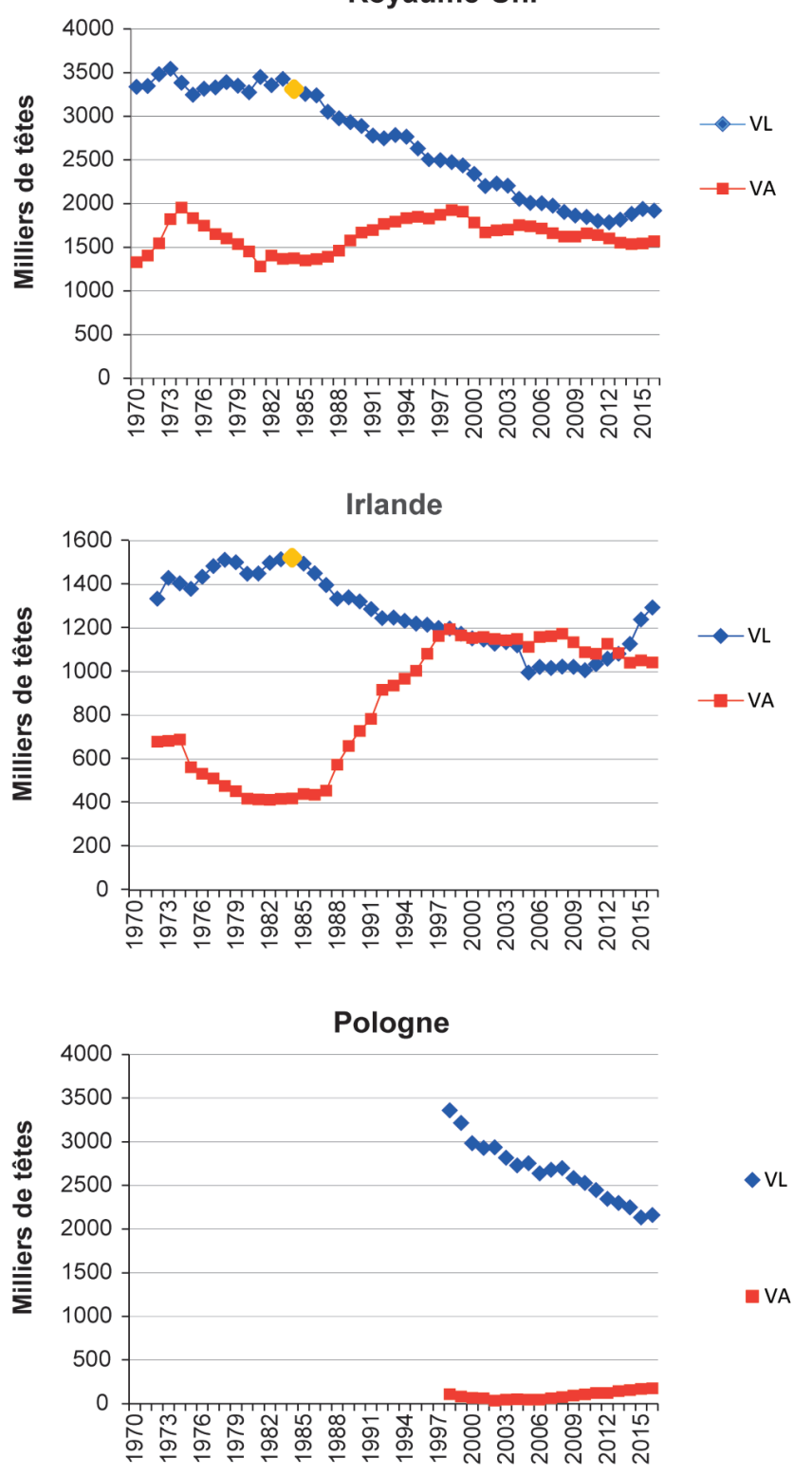

\section{2 / Principales caractéristiques des systèmes spécialisés Européens «bovin élevage et engraissement »}

Les systèmes spécialisés « bovin élevage et engraissement $»^{3}$, producteurs de viande bovine (BV) représentent $4 \%$ des exploitations agricoles de l'UE à 28 (UE28) et utilisent 9\% de la SAU avec $13 \%$ des UGB (tableau 3). Elles exploitent en moyenne 40 ha dont $7 \%$ consacrés aux céréales et $91 \%$ à la surface fourragère. Le troupeau moyen compte 42 UGB dont 19 vaches. Le chargement moyen est de 1,14 UGB/ha SFP, cette dernière étant composée de $3 \%$ de maïs, $1 \%$ de légumineuses, $15 \%$ de prairies temporaires et $80 \%$ de prairies perma- nentes. Elles sont gérées par 1,14 UTA dont 0,18 salarié, ce qui fait en moyenne 35 ha et 37 UGB par travailleur.

La France compte $10 \%$ de ses exploitations dans ce système. Elles exploitent $12 \%$ de la SAU et représentent $16 \%$ des UGB nationales. Avec 70 ha de SAU composée de $8 \%$ de céréales et $92 \%$ de SFP, ce sont elles qui représentent la taille moyenne la plus importante. Le troupeau composé de 42 VA atteint 73 UGB. Le système fourrager est peu intensif : le chargement moyen est identique $(1,13$ $\mathrm{UGB} / \mathrm{ha}$ ) à la moyenne de l'UE28 ainsi que la part de maïs (3\%), avec en revanche plus de prairies temporaires $(24 \%)$. Ces exploitations sont gérées par 1,17
UTA vs 1,14 pour la moyenne de l'UE, dont $33 \%$ vs $14 \%$ est salarié.

L'Espagne ne compte que 5\% de ses exploitations dans ce système, mais elles occupent, de manière similaire à la France, $11 \%$ de la SAU avec $17 \%$ des UGB. Ces exploitations sont structurées essentiellement autour de prairies permanentes $(95 \%)$ avec peu de cultures $(5 \%)$. En moyenne elles sont gérées par 1,13 UTA pour 50 ha, 27 vaches et 47 UGB. Le système fourrager de ces exploitations espagnoles est le plus extensif de l'Europe à base de prairies permanentes qui nourrissent $1,01 \mathrm{UGB} /$ ha avec très peu de maïs $(0,5 \%)$ et de prairies temporaires $(2 \%)$.

${ }^{3}$ OTEX ou TF « Type Farm » n46 « spécialisée bovin élevage et engraissement ». 
Tableau 3. Caractéristiques des exploitations spécialisées « bovin élevage et engraissement » (OTEX 46) en 2013 dans l'Union Européenne (UE28) (d'après Eurostat).

\begin{tabular}{|c|c|c|c|c|c|c|}
\hline & UE28 & France & Espagne & $\begin{array}{l}\text { Royaume- } \\
\text { Uni }\end{array}$ & Irlande & 24 autres \\
\hline Nombre d'exploitations & 390830 & 48860 & 51830 & 33920 & 78760 & 177460 \\
\hline$\%$ de l'ensemble des exploitations du pays & 4 & 10 & 5 & 18 & 56 & 2 \\
\hline SAU moyenne (ha) & 40 & 70 & 50 & 61 & 27 & 31 \\
\hline$\%$ de l'ensemble de la SAU du pays & 9 & 12 & 11 & 12 & 43 & 5 \\
\hline Ha Céréales ( $\%$ SAU) & 6,6 & 7,8 & 4,6 & 4,8 & 1 & 9,7 \\
\hline Ha SFP (\% SAU) & 91 & 92 & 92 & 94 & 99 & 87 \\
\hline \multicolumn{7}{|l|}{ Composition de la SFP (\%SFP) } \\
\hline Maïs Fourrage & 3 & 3 & 0,5 & 1 & 0 & 6 \\
\hline Légumineuse & 1 & 1 & 0,5 & 0 & 0 & 2 \\
\hline Prairie Temporaire & 15 & 24 & 2 & 15 & 14 & 15 \\
\hline Prairie Permanente & 80 & 72 & 95 & 84 & 86 & 73 \\
\hline Nombre d'UTA (Travail Annuel) & 1,14 & 1,17 & 1,13 & 1,16 & 1,11 & 1,15 \\
\hline dont Salariées & 0,18 & 0,41 & 0,24 & 0,13 & 0,07 & 0,15 \\
\hline \% de Main-d'œuvre familiale & 86 & 67 & 80 & 90 & 95 & 89 \\
\hline Nombre d'UGB & 42 & 73 & 47 & 71 & 31 & 31 \\
\hline$\%$ de l'ensemble des UGB du pays & 13 & 16 & 17 & 18 & 41 & 7 \\
\hline \multicolumn{7}{|l|}{$\begin{array}{l}\text { Composition du troupeau } \\
\text { (Nombre de têtes par exploitation) }\end{array}$} \\
\hline Nombre de Vaches Allaitantes & 19 & 42 & 27 & 24 & 12 & 12 \\
\hline Nombre de têtes bovin $<1$ an & 23 & 39 & 20 & 29 & 13 & 22 \\
\hline Nombre de têtes mâles $1-2$ ans & 7 & 3 & 5 & 16 & 7 & 7 \\
\hline Nombre de têtes femelles $1-2$ ans & 7 & 12 & 6 & 14 & 6 & 5 \\
\hline Nombre de têtes mâles $>2$ ans & 2 & 2 & 2 & 6 & 4 & 1 \\
\hline Nombre de têtes femelles $>2$ ans & 4 & 11 & 5 & 6 & 3 & 3 \\
\hline Total Nombre de têtes bovins & 63 & 110 & 67 & 95 & 44 & 50 \\
\hline Nombre de brebis & 6 & 5 & 4 & 33 & 4 & 2 \\
\hline Chargement UGB/Ha SFP & 1,14 & 1,13 & 1,01 & 1,23 & 1,16 & 1,16 \\
\hline SAU/UTA & 35 & 60 & 44 & 53 & 24 & 27 \\
\hline UGB/UTA & 37 & 62 & 41 & 61 & 28 & 27 \\
\hline
\end{tabular}

Le Royaume-Uni même avec un cheptel allaitant stable compte $18 \%$ des exploitations en système BV. Celles-ci n'utilisent que $12 \%$ de la SAU avec $18 \%$ des UGB du pays. Ces fermes sont relativement grandes, quoique légèrement moins qu'en France. Sur les 61 ha exploités en moyenne, seulement $5 \%$ sont cultivées en céréales, la SFP ( $95 \%$ de la SAU) est constituée de $84 \%$ de prairies permanentes, $14 \%$ de de prairies temporaires et seulement de $1 \%$ de maïs fourrage. Ces surfaces nourrissent un important cheptel de 71 UGB, composé de 24 vaches allaitantes et leur suite, avec en particulier un nombre de mâles âgés et une troupe ovine (33 brebis) associée plus impor- tants qu'en France. Le chargement (1,23 $\mathrm{UGB} / \mathrm{ha}$ ) est un peu plus élevé qu'ailleurs, alors que la main-d'œuvre est de même niveau avec peu de salariés.

L'Irlande se singularise par l'importance de ce système de production qui concerne $56 \%$ des exploitations, $43 \%$ de la surface et $41 \%$ des UGB, et par la faible taille de ses structures : 27 ha SAU et 31 UGB (12 VA et leur suite composée comme au RU des élèves mâles en croissance ou finition) pour une main-d'œuvre voisine de celle des 24 autres pays avec 1,11 UTA $^{4}$ à $95 \%$ familiale. Le niveau de chargement de la SFP est également moyen (1,16 UGB/ha
SFP) et identique à la moyenne des 24 autres pays. La SFP est composée exclusivement de prairies dont $86 \%$ sont permanentes sans cultures ni fourragères ni céréalières (1\% de la SAU).

Dans l'ensemble des 24 autres pays sont élevées 29\% des VA de l'UE28, les systèmes BV correspondent à $2 \%$ des exploitations de ces pays. Ces fermes utilisent 5\% de la SAU et élèvent 7\% des UGB. Elles sont de petite taille avec 31 ha SAU et 31 UGB (12 VA). Elles ont généralement moins de prairies et plus de cultures fourragères ( $6 \%$ de maiis) et céréalières $(10 \%)$ pour un chargement quasiment identique $(1,16 \mathrm{UGB} / \mathrm{ha})$.

\footnotetext{
${ }^{4}$ Une Unité de Travail Annuel (UTA) correspond au travail d'une personne employée dans une exploitation agricole à temps plein. Dans le cas de pluriactif c'est-à-dire avec une activité hors exploitation, situation fréquente dans ce pays, seule la part du travail agricole est estimée.
} 


\section{3 / Des surfaces et des troupeaux en augmentation sauf en Irlande et un chargement plutôt en réduction}

L'emprise territoriale des systèmes BV dans l'UE 28 s'est accrue passant de 14,5 millions d'ha SAU en 2005 à 15,7 en 2013 malgré un fléchissement récent (16 millions en 2010). La part de la SAU européenne exploitée par les exploitations BV s'accroît ainsi de 8,4 à $9,0 \%$ entre 2005 et 2013. Sur cette période, le nombre total d'exploitations a diminué de $25 \%$ alors que les systèmes BV en ont perdu seulement $5,6 \%$. Leur importance s'accroit donc passant de 2,9 à $3,6 \%$, mais reste quand même très limitée à l'échelle de l'UE. Leur main-d'œuvre a légèrement diminué de 1,17 à 1,14 UTA, alors que la SAU moyenne a augmenté de 35 à 40 ha. La surface exploitée par travailleur est donc passée de 30 à 35 ha, soit $+17 \%$, et le cheptel de 33,8 à 36,6 UGB / UTA, soit + 8\% (figure 6). Le chargement a diminué de 1,25 à 1,14 UGB/ha SFP, sans entrainer de modification significative dans la composition de la SFP : de 2,8 à 2,9\% de maïs fourrage, de 15 à $14,5 \%$ de prairies temporaires et toujours $80 \%$ de STH.

Les fermes françaises de structure déjà importante sont celles dont la taille a le plus augmenté (+ 24\% en SAU/UTA) avec une accélération entre 2010 et 2013 $(+3,5 \% /$ an $)$ et ceci avec une faible variation de main-d'œuvre de 1,13 à 1,17 UTA. Ces agrandissements se sont accompagnés d'une baisse de $35 \%$ du nombre d'exploitations dans ce système (de 75 à 49 000) alors que la diminution des effectifs globaux des exploitations agricoles a été de - $17 \%$. Le RU connaît une évolution similaire mais avec un ralentissement récent. Les cheptels de ces deux pays sont importants et en croissance pour atteindre 61 UGB/UTA même si cet accroissement se ralentit sur la période récente au RU.

Ces tailles de cheptel sont près de deux fois celles observées en Espagne qui pourtant augmentent de 30 à 40 UGB/UTA. En surface et par travailleur les systèmes espagnols passent de 32 à 44 ha SAU/UTA. C'est dans ce pays que la main-d'œuvre a le plus diminué de 1,30 à 1,13 UTA. La productivité du travail tend donc à s'harmoniser avec celle de la France.

En revanche en Irlande, l'évolution de la taille des structures est particulière en lien avec le peu d'alternative d'emplois industriels de ce pays et le prix des terres. Non seulement elles n'augmentent pas, mais elles diminuent même : - 1 ha SAU et surtout $-6,7$ UGB avec encore un peu plus de main-d'œuvre (1,05 à 1,11 UTA).

Figure 6. Évolution entre 2005 et 2013 de la taille des exploitations et des troupeaux par Unité de Travail Homme (UTH) et du chargement de la surface fourragère dans les exploitations spécialisées " bovins viande » (d'après Eurostat).

Taille de l'exploitation (SAU / UTH)

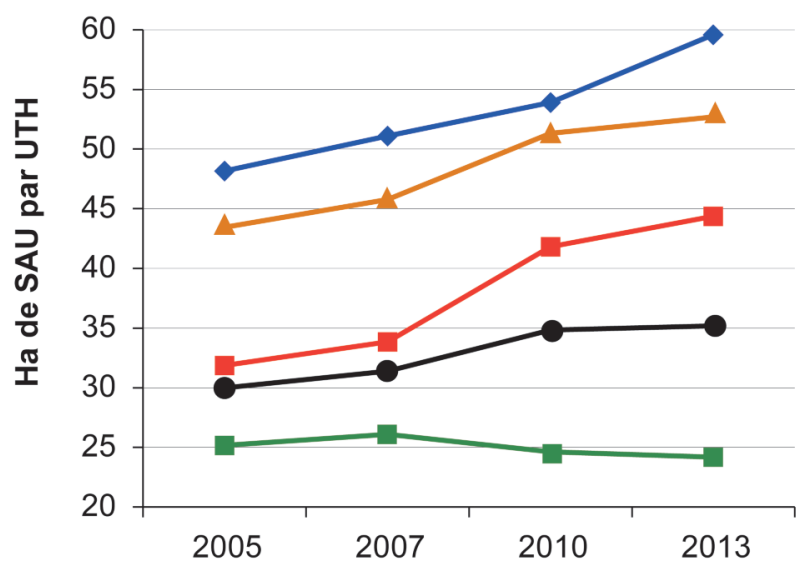

Taille du troupeau (UGB / UTH)



Chargement de la surface fourragère

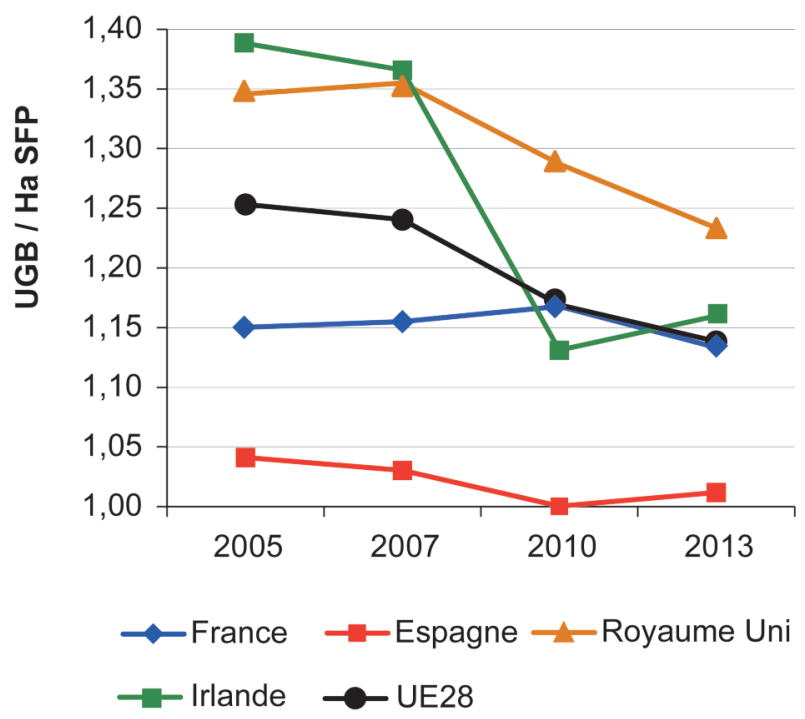

Vu l'évolution divergente des surfaces et des cheptels, les chargements se réduisent significativement au RU (de 1,35 à $1,23 \mathrm{UGB} / \mathrm{ha} \mathrm{SFP}$ ) et en Irlande (de 1,39 à 1,16$)$ et sont quasiment stables en
France $(1,15$ à 1,13$)$ et en Espagne $(1,04$ à 1,01) (figure 6). Le système fourrager est globalement peu modifié, en particulier en Espagne. En Irlande, la STH augmente de 5 points (de 80,6 à $85,7 \%$ ) au 
détriment des Prairies Temporaires (PT), le maïs restant absent $(0,1 \%)$. En France au contraire, la part des surfaces en PT augmente (de 20,6 à 23,6\%) au détriment de la STH, la part de maïs restant stable (3,3 à 3,4\%). De même au RU, la part des PT augmente de 12,7 à 15,2\%, remplaçant de la STH, le maïs fourrage passe de 0,4 à $0,8 \%$ de la SFP.

\section{3 / Analyse comparée des performances économiques des exploitations}

\section{1 / Les données utilisées}

Les données économiques mobilisées sont fournies par le RICA européen («Farm Accountancy Data Network $(F A D N) »)$. Elles concernent les exploitations dites " moyennes et grandes " correspondant à l'ancienne dénomination « professionnelles », c'est-à-dire celles tirant la totalité ou l'essentiel de leur revenu de l'activité agricole. Nous considèrerons dans cette partie l'orientation technico-économique (OTEX ou TF « Type Farm ») n॰ 49 « spécialisée bovin » («cattle ») » qui inclut, outre le $\mathrm{TF} \mathrm{n}^{\circ} 46$ « spécialisée bovin élevage et engraissement » que nous avons utilisé dans la partie précédente, le $n^{\circ} 47$ « mixte bovin lait et viande ». Les caractéristiques de cet échantillon sont rassemblées dans le tableau 4. Leurs structures sont donc par définition plus grandes.

Les exploitations « petites » (ni moyennes ni grandes) sont souvent de microexploitations ( 1,7 ha en moyenne à l'échelle de l'UE) compte-tenu de la définition des seuils minimaux très bas et variables selon les pays (ainsi en France, on considère comme unité exploitation une entité ayant une gestion indépendante et qui met en valeur au moins 1 ha SAU ou une vache ou 5 brebis ou 20 ares de cultures spécialisées...). En 2013, les exploitations « moyennes et grandes » analysées par la suite représentent $46 \%$ du total des exploitations européennes, tous systèmes de production confondus, mais elles exploitaient, en $2013,94 \%$ de la SAU avec $81 \%$ de la main-d'œuvre et $89 \%$ des UGB.

\section{2 / Des moyens de production importants en France et au RU, faibles en Irlande et dans les 24 autres pays d'Europe}

Comme pour les observations de la partie précédente, il apparaît nettement que la France et le RU disposent des plus grandes structures Ainsi au même niveau de chargement (1,23 UGB/ha SFP), il y a en France 109 ha et 123 UGB pour 1,51 UTA et 113 ha et 131 UGB pour
1,48 UTA au RU (tableau 4). En complément des bovins viande, les exploitations du RU élèvent une troupe ovine de 21,5 UGB (taille moyenne), et celles de France 9 vaches laitières tout en cultivant $10 \%$ de la SAU en céréales. En Espagne, les exploitations présentent une taille de 60 ha et 46 UGB pour 1,22 UTA, conduits très extensivement avec $0,75 \mathrm{UGB} / \mathrm{ha}$ SFP. En Irlande et dans les 24 autres pays, les exploitations sont en moyenne plus petites avec 44 et 37 ha SAU, 50 et 36 UGB pour 1,10 et 1,42 UTA et un chargement de 1,16 et 1,09 UGB/ha SFP.

On notera que le fermage qui est le mode de faire valoir majoritaire représente $54 \%$ des surfaces dans ces systèmes à l'échelle de l'UE. Ce mode de faire valoir est moins développé en Irlande et au RU (16 et $38 \%$ respectivement) alors qu'il représente $84 \%$ de la SAU en France.

En Espagne, les exploitations mettent en ouvre moins de capital par ha SAU (presque 50\% de moins que la moyenne). Du fait du faible chargement, il y a moins d'animaux par ha donc moins de capital cheptel, très peu de capital sous forme de matériel et peu de bâtiments.

La valeur financière du cheptel est voisine de la moyenne de $1158 €$ par UGB partout sauf en France, où ce montant est supérieur de 18\% (1 $372 € / \mathrm{UGB})$. La valeur du troupeau constitue $55 \%$ du capital d'exploitation en France, 57\% au RU et $40 \%$ en moyenne UE. Les exploitations du RU disposent de bâtiments peu importants et déjà en partie amortis, du fait d'exploitants qui renouvellent peu leurs investissements. Dans les 24 autres pays les exploitations sont particulièrement bien équipées en matériels et installations, et le cheptel ne représente chez eux que $28 \%$ du capital fixe d'exploitation.

L'endettement est conséquent en France où $32 \%$ du capital fixe d'exploitation est emprunté alors qu'il est plus faible ailleurs, particulièrement en Espagne et Irlande où il n'en représente que $2 \%$ (tableau 4). Ce chiffre, qui correspond à des investissements très modestes et qui ne sont pas renouvelés, interroge sur la pérennité à terme de ces exploitations.

\section{3 / Des itinéraires variés et des résultats économiques contrastés}

Au niveau de l'atelier « animal » (principalement bovin viande avec parfois un peu de lait ou d'ovin), les exploitations françaises obtiennent un produit par UGB du niveau moyen de l'UE avec des charges opérationnelles modérées en particulier sur le poste " aliment concentré ». Ceci s'explique par la pré- sence, dans notre pays, d'une majorité $(74 \%)$ de producteurs «naisseurs » situés dans le grand Massif central ou sud-ouest qui commercialisent surtout des jeunes animaux destinés à un engraissement rapide en ateliers spécialisés (Bébin et al 1995). Les exploitations irlandaises ont aussi des charges réduites, mais obtiennent un faible produit, avec des animaux croisés engraissés à l'herbe plutôt légers. En Espagne et au RU, les exploitations ont aussi des produits faibles, mais avec un peu plus de charges. Les 24 autres pays dégagent en moyenne un produit plus élevé mais avec beaucoup d'intrants en particulier en utilisation de concentrés. En définitive, la France dispose des ateliers les plus performants avec $542 € / \mathrm{UGB}$ de marge animale, à l'inverse du RU et de l'Espagne avec 226 et $254 € / \mathrm{UGB}$. La marge en Irlande est intermédiaire mais faible avec $302 € / \mathrm{UGB}$.

Si les comparaisons sont réalisées par ha de $S A U$, en Espagne, les exploitations combinent une faible production par UGB et un chargement limité. Leur produit est alors de $639 €$ par ha, résultat obtenu avec très peu de charges de structure, seulement $52 € /$ ha d'amortissement des équipements et très peu de facteurs externes $(59 € /$ ha vs 136 en moyenne UE) qui comprennent fermages, coûts salariaux et frais financiers. Le prix du fermage dans ce pays est particulièrement modeste $(52 € /$ ha $)$ du fait de la qualité médiocre des terres. Le coût du travail salarié reste assez faible (14 K€/UTA salarié).

De même en Irlande, le produit est faible avec $893 € /$ ha malgré un chargement supérieur, avec un niveau d'amortissements de $153 € /$ ha. Le montant des facteurs externes (salaire, fermage, frais financiers) est limité à $65 € /$ ha soit guère plus que l'Espagne en raison de la faible part de terres en fermage (16\%), du peu de main-d'œuvre salariée ( 0,03 UTA) et d'endettement $(2 \%)$. Pourtant dans ce pays, le coût d'usage de la terre est très élevé $226 € /$ ha, soit 4 fois plus que l'Espagne et deux fois le montant moyen de l'UE (110 €/ha). Le taux moyen d'intérêt $5,6 \%$ est aussi très supérieur $(3,5 \%)$ et les coûts salariaux sont élevés $20 \mathrm{~K} € /$ UTA salarié.

$\mathrm{Au} \mathrm{RU}$, pour un produit moyen de $1115 € /$ ha, les exploitations utilisent beaucoup de consommations intermédiaires $(955 € /$ ha) et peu d'équipements (amortissements de $180 € /$ ha). Les coûts salariaux sont maximums avec près de $24 \mathrm{~K} € / \mathrm{UTA}$ salarié, ce qui entraine $120 € /$ ha en montant de facteurs externes.

En France, les résultats des exploitations sont limités par les charges de structure : $253 € /$ ha d'amortissement et $152 € /$ ha de facteurs externes provenant moins 
Tableau 4. Caractéristiques et résultats des exploitations " moyennes et grandes » "spécialisées bovin » (TF14 = 49) dans I'Union Européenne (UE28) en 2013 (d'après RICA européen).

\begin{tabular}{|c|c|c|c|c|c|c|}
\hline & UE28 & France & Espagne & Royaume-Uni & Irlande & 24 autres \\
\hline Nombre d'exploitations & 373360 & 41370 & 45250 & 18810 & 43210 & 224720 \\
\hline Nombre d'UTA & 1,37 & 1,51 & 1,22 & 1,48 & 1,10 & 1,42 \\
\hline dont Salariées & 0,14 & 0,12 & 0,12 & 0,23 & 0,03 & 0,16 \\
\hline SAU moyenne (ha) & 52 & 109 & 60 & 113 & 44 & 37 \\
\hline$\%$ Ha en fermage & 54 & 84 & 52 & 38 & 16 & 51 \\
\hline Ha Céréales (\% SAU) & 9 & 10 & 5 & 6 & 1 & 13 \\
\hline Nombre d'UGB Bovin & 51 & 121 & 45 & 109 & 48 & 35 \\
\hline dont Vaches Laitières & 3.5 & 9 & 0.3 & 2.4 & 1.3 & 3.6 \\
\hline Nombre d'UGB Ovin & 1.9 & 1.3 & 0.5 & 21.5 & 2.2 & 0.6 \\
\hline Chargement UGB/Ha SFP & 1,08 & 1,23 & 0,75 & 1,23 & 1,16 & 1,09 \\
\hline Capital fixe d'exploitation (€/UGB) & 2866 & 2504 & 2025 & 2019 & 2537 & 3648 \\
\hline dont cheptels & 1158 & 1372 & 1162 & 1155 & 1129 & 1032 \\
\hline dont matériels & 714 & 569 & 300 & 551 & 475 & 1022 \\
\hline dont bâtiments & 994 & 563 & 564 & 313 & 933 & 1594 \\
\hline Capital fixe d'exploitation ( $€ /$ ha SAU) & 2921 & 2810 & 1548 & 2338 & 2921 & 3578 \\
\hline dont cheptels & 1180 & 1540 & 888 & 1338 & 1300 & 1012 \\
\hline dont matériels & 728 & 638 & 229 & 638 & 547 & 1003 \\
\hline dont bâtiments & 1013 & 632 & 431 & 362 & 1074 & 1564 \\
\hline Taux d'endettement (\%) & 11 & 32 & 2 & 8 & 2 & 13 \\
\hline Subventions totales (€/expl.) & 20841 & 42192 & 14131 & 37482 & 19077 & 17208 \\
\hline$(€ /$ ha SAU) & 401 & 387 & 236 & 332 & 434 & 465 \\
\hline dont ICHN (€/ha SAU) & 43 & 39 & 16 & 24 & 48 & 58 \\
\hline dont DPU (€/ha SAU) & 222 & 215 & 104 & 248 & 302 & 235 \\
\hline Subventions totales (€/UTA) & 15212 & 27942 & 11583 & 25326 & 17343 & 12118 \\
\hline Subventions (\% Produit total) & 26 & 27 & 27 & 23 & 23 & 25 \\
\hline Subventions (\% Revenu Net) & 129 & 203 & 80 & 172 & 151 & 114 \\
\hline Produit animal (€/UGB) & 809 & 800 & 699 & 696 & 635 & 876 \\
\hline Charges animales (€/UGB) & 406 & 258 & 445 & 470 & 333 & 434 \\
\hline dont aliments concentrés (€/UGB) & 337 & 208 & 400 & 380 & 275 & 404 \\
\hline Marge animales (€/UGB) & 403 & 542 & 254 & 226 & 302 & 442 \\
\hline Produit total hors aides ( $€ /$ ha SAU) & 1131 & 1039 & 639 & 1115 & 893 & 1399 \\
\hline $\begin{array}{l}\text { Consommations } \\
\text { intermédiaires (€/SAU) }\end{array}$ & 868 & 823 & 476 & 955 & 789 & 1016 \\
\hline Amortissements (€/SAU) & 209 & 253 & 52 & 180 & 153 & 256 \\
\hline $\begin{array}{l}\text { Valeur Ajoutée Nette hors aides } \\
(€ / S A U)\end{array}$ & 54 & -37 & 111 & -20 & -49 & 127 \\
\hline Fermage $(€ /$ ha $)$ affermées & 110 & 112 & 52 & 114 & 226 & 120 \\
\hline Salaires + charges (€/UT) Salariés & 16043 & 18083 & 14142 & 23870 & 20333 & 14961 \\
\hline Intérêt moyen des emprunts (\%) & 3,5 & 2,9 & 3,6 & 3,2 & 5,6 & 3,7 \\
\hline Revenu Brut d'exploitation $^{(1)}(€ /$ UTA) & 24763 & 42634 & 19849 & 37157 & 20605 & 21646 \\
\hline $\begin{array}{l}\text { Valeur Ajoutée Nette avec aides } \\
\text { subventions ( }(/ \text { UTA) }\end{array}$ & 16716 & 24346 & 17237 & 23464 & 14528 & 15062 \\
\hline Revenu $\left.\mathrm{Net}^{(2)} / \mathrm{ha} \mathrm{SAU}\right)$ & 307 & 190 & 295 & 192 & 290 & 409 \\
\hline Revenu Net (€/UTF) & 12849 & 14972 & 15543 & 17465 & 11799 & 11731 \\
\hline
\end{tabular}

UTA : Unité de Travail Annuel ; SAU : Surface Agricole Utile ; UGB : Unité Gros Bovin, SFP : Surface Fourragère Principale ; ICHN : Indemnité Compensatoire de Handicap Naturel ; DPU : Droit à Paiement Inique ; UTF : Unité de Travail Familial.

(1) $:$ EBE + Salaires + Fermages $=$ Valeur Ajoutée brute $=$ Produit + Subventions - consommations intermédiaires.

(2) : Représente la rémunération des facteurs fixes de production (travail, terre et capital) de l'exploitation et la rémunération du risque de l'entrepreneur (perte/profit), pour l'année comptable. 
des coûts unitaires de fermage $112 € /$ ha ou des capitaux empruntés $(2,9 \%$ d'intérêt) ou des coûts salariaux (18 K€/UTA salarié) que du fait que $84 \%$ du foncier est en fermage et $32 \%$ du capital est emprunté donc soumis à frais financiers.

La Valeur Ajoutée Nette hors subventions se révèle être négative dans 3 pays : la France, le RU et l'Irlande, c'est-àdire que le produit sans subventions ne couvre pas les charges réelles engagées (consommations intermédiaires + amortissements). Cela montre l'importance des soutiens directs pour la viabilité de ces systèmes qui avaient été institués pour compenser la baisse des prix voulue par la PAC de 1992. Ces soutiens ont depuis atteint des montants importants par exploitation ou UTA en France et au RU car très liés à leurs tailles. Par ha, ils sont maximums en Irlande et dans les 24 autres pays, et sont légèrement audessous de la moyenne de l'UE en France (387 vs $401 € /$ ha SAU). Le RU et surtout l'Espagne bénéficient de montants de soutiens très inférieurs, avec 332 et $236 € /$ ha respectivement. Historiquement en Espagne, les Droits à Paiements Uniques ne sont que de 104 vs $222 € /$ ha pour la moyenne de l'UE. Ce pays, pourtant en conditions difficiles, n'a que peu d'Indemnités Compensatoires d'Handicaps Naturels permanents (ICHN), avec 16 vs $43 € /$ ha pour la moyenne de l'UE. Ces subventions n'y constituent alors « que » $80 \%$ du revenu net (et $27 \%$ du produit total avec aides). En France elles se montent par UTA à $28 \mathrm{~K} €$ pour seulement $15 \mathrm{~K} €$ de revenu net tout en ne constituant que $27 \%$ du produit, niveau voisin dans tous les pays.

Raisonné à l'échelle de l'exploitation et ramené à la main-d'œuvre (par UTA), le Revenu Brut ${ }^{5}$ c'est-à-dire l'Excédent Brut d'Exploitation (EBE) avant déductions des coûts salariaux et fermages, est en France et au RU de 42 et $37 \mathrm{~K} € /$ UTA. Il est élevé par rapport à tous les autres pays où il n'atteint que 20-21 K€/UTA. C'est essentiellement lié à la bonne productivité du travail de ces pays comme cela a été relevé précédemment.

Du fait des niveaux d'amortissements et des facteurs externes (salaire, fermage, frais financiers) très différents d'un pays à l'autre, les Revenus Nets ${ }^{6}$ par UT Familial (UTF) sont en définitive assez voisins dans les différents pays analysés. Il se monte à $15 \mathrm{~K} €$ en France, niveau proche de celui de l'Espagne alors qu'il est un peu supérieur au RU avec $17 \mathrm{~K} € /$ UTF. En Irlande et dans les 24 autres
Figure 7. Évolution comparée du revenu courant par Unité de Travail Familial (k€/UTF) de l'ensemble des systèmes de production (Tot) et des systèmes "spécialisés bovins » (OTEX 49 BV) en France et dans I'UE (Source RICA Européen).

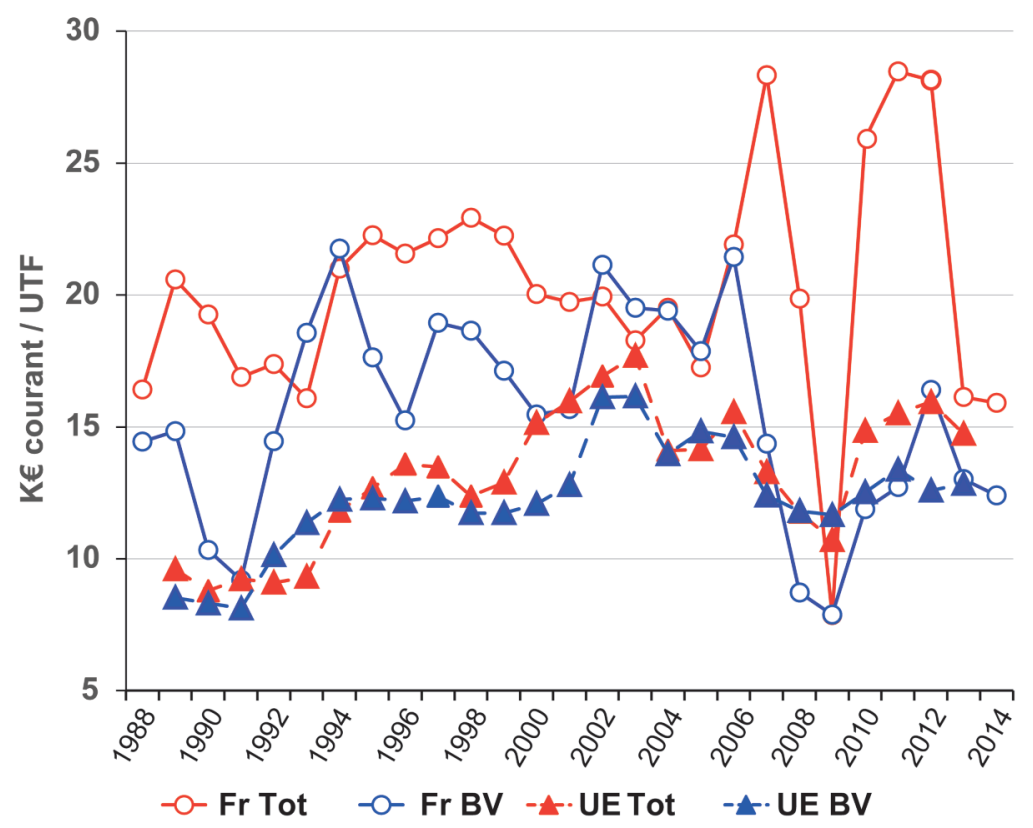

pays, les revenus nets sont sensiblement inférieurs (12 K€/UTF).

Cette comparaison met en évidence des choix différents entre les pays selon leurs contextes. Les élevages du RU et de France ont choisi la voie de la productivité du travail, mais elle s'accompagne de charges d'équipement les plus élevées. Cela illustre bien la substitution main-d'œuvre - équipements qui se retrouve moins dans les autres pays notamment en Espagne et en Irlande. Sur le plan économique, cela se traduit par des EBE nettement plus importants mais avec peu d'impact final sur le Revenu Net (RCAI ${ }^{6}$ ) de l'éleveur. L'EBE supplémentaire dégagé par l'agrandissement et l'augmentation de la productivité du travail, sert essentiellement à couvrir les charges foncières, financières et d'amortissements supplémentaires. Il faudrait cependant nuancer cet effet de la taille en fonction des situations très contrastées dans les régions.

L'analyse temporelle qui suit permet cependant de mieux comprendre les déterminants de ces situations nationales.

\section{4 / Des revenus nets qui stagnent depuis 2000 dans tous les pays}

Les exploitations européennes dont l'orientation technico-économique (OTEX) est « spécialisée bovin » (TF14 $=49$ ) dégagent un revenu net (RCAI) plutôt faible par rapport à la moyenne générale, particulièrement depuis 2010 (figure 7). Ce constat se vérifie aussi pour la France de façon encore plus nette : depuis 1989, le revenu net des exploitations BV n'a jamais été supérieur à la moyenne des revenus de l'ensemble des exploitations. En monnaie constante, déflatée de l'augmentation moyenne des prix à la consommation, le niveau de revenu net par travailleur familial (UTF) se retrouve en $2013 \mathrm{au}$ niveau de 1989-1991 et ce malgré les gains de productivité du travail déjà relevés précédemment (Veysset et al 2015).

Si l'on reprend les résultats issus du RICA européen depuis 1989, les revenus des éleveurs augmentaient en monnaie courante dans tous les pays avant l'année 2000, sans que les classements relatifs des pays ne soient modifiés. Les exploitations françaises bénéficiaient grâce à la taille de leurs structures d'un revenu net relativement ${ }^{7}$ élevé (figure $8 \mathrm{~A}$ ) surtout de 1993 à 2002. Depuis 2002 ce revenu semble stagner partout et sa variabilité s'est accrue après la crise de 2008. En France, il a décroché fortement tout en restant supérieur à la moyenne de l'UE, sauf en 2009 (Lherm et al 2015). Les exploitations du RU et de l'Espagne ont plutôt amélioré leurs positionnements en comprimant fortement leurs dépenses en intrants et investissements. Elles ont donc moins subi les crises liées à la

\footnotetext{
5 Équivalent aux produits avec primes diminués des consommations intermédiaires.

${ }^{6}$ Sur une campagne, le Produit - Charges réelles, correspond au RCAI (Résultat Courant Avant Impôts ou anciennement appelé Revenu Agricole Familial). ${ }^{7}$ À relativiser par rapport à l'ensemble des exploitations nationales (figure 7). On rappellera également que les charges sociales familiales ne sont pas déduites car considérées comme privées.
} 
flambée des prix de la plupart des intrants consécutifs à l'envolée du prix du pétrole (2007-2010) et/ou de celui des cours mondiaux des céréales et des tourteaux. Cette situation est liée à leur choix de faibles dépenses de fonctionnement et d'investissements. En revanche, en Irlande, les exploitations restent handicapées par leurs plus petites structures, même si certaines années ont été plus favorables comme 2008, 2011 et 2012.

L'analyse de quelques composantes du revenu permet de mieux comprendre le recul relatif des exploitations françaises. Il ne provient pas d'une moindre maîtrise de l'atelier « animal » dont la marge depuis 1995 est constamment nettement audessus de l'ensemble des autres pays (figure 8B) comme nous l'avions déjà constaté dans l'analyse de l'année 2013 (cf. § 3.3). Le montant de l'EBE avant déductions des coûts salariaux et fermages est, lui aussi, constamment meilleur et a progressé de $119 \%$ en monnaie courante par UTA sur la période (figure 8C). Mais ce recul relatif provient surtout de la conséquence des investissements en équipements, encouragés par des mesures

Figure 8. Évolution des performances économiques des systèmes « spécialisés bovins » (OTEX 49 BV) dans l'UE (Source RICA européen) : A) Revenu Net par Unité de Travail Familial (en € courant / UTF) ; B) Marge animale par unité de gros bovin (en $€$ courant /UGB) ; C) Excédent Brut d'Exploitation par Unité de Travail annuel (€ courant / UTA) ; D) Montant des amortissements par Unité de Travail Annuel (en € courant/UTA).

\section{A) Revenu net par unité de travail familial}

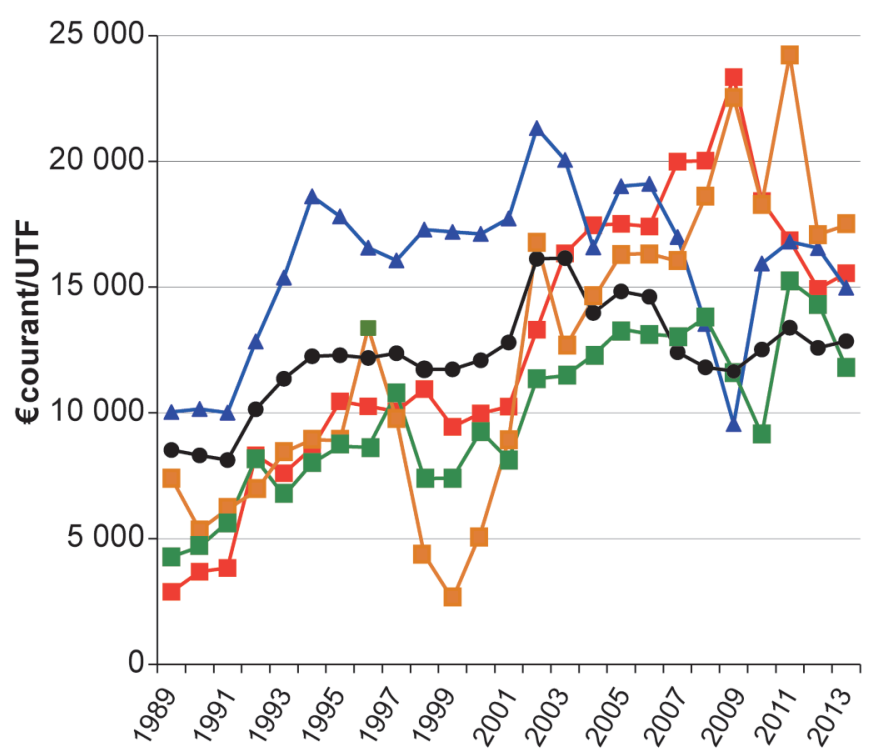

C) Excédent brut d'exploitation par unité de travail annuel

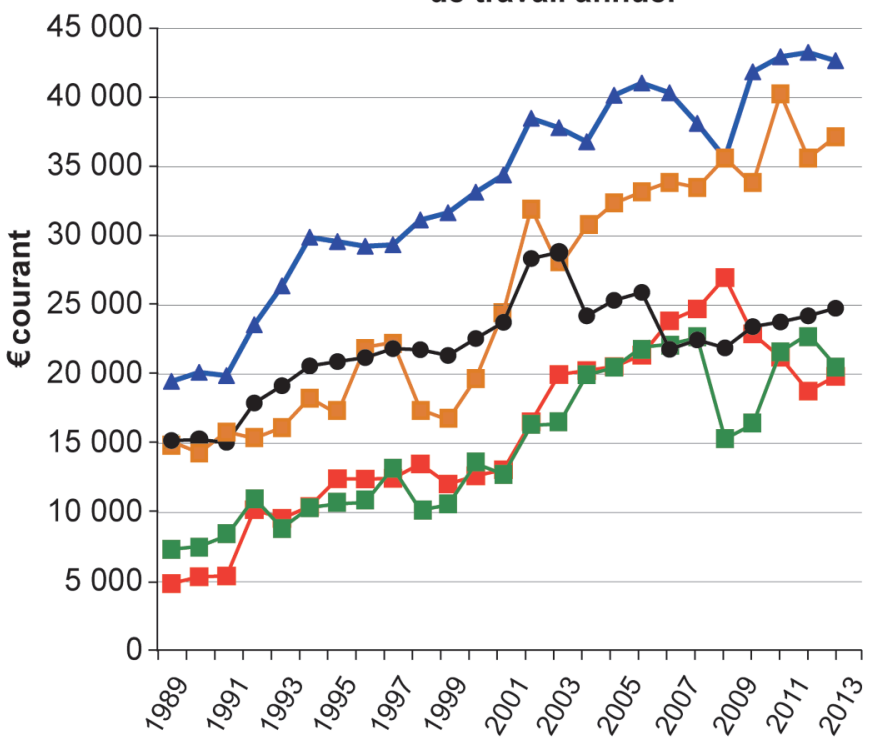

B) Marge animale par unité de gros bétail

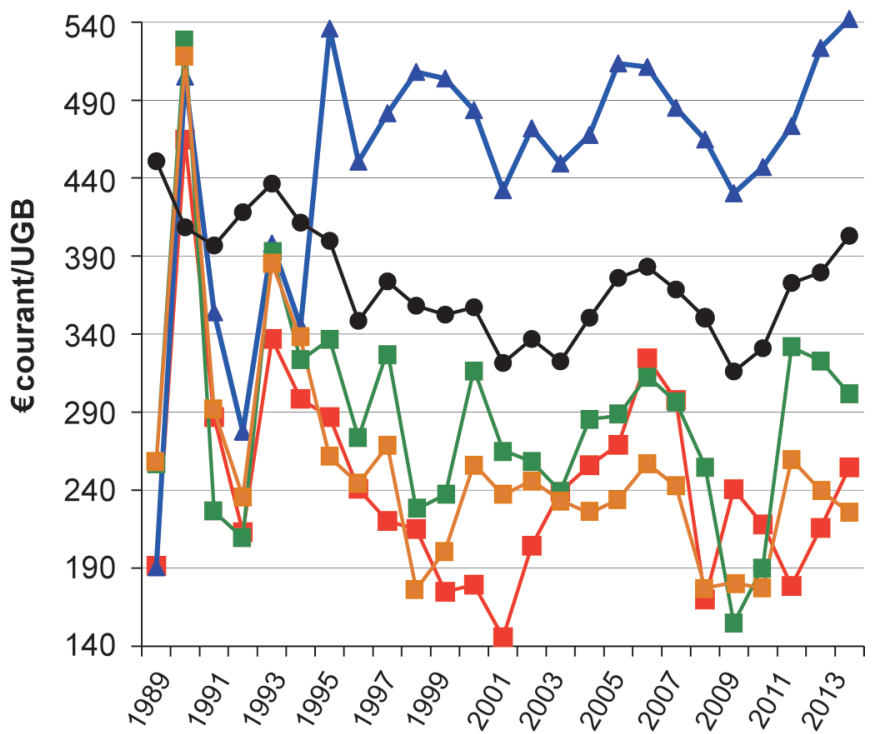

D) Montant des amortissements par unité de travail annuel

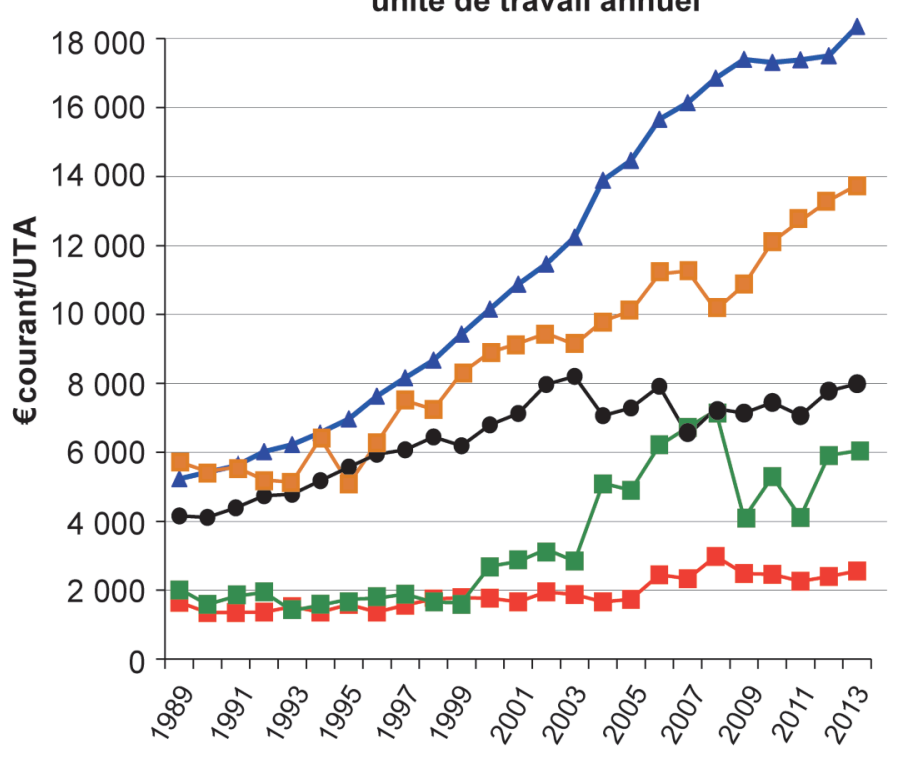


de défiscalisation, pour assumer la meilleure productivité du travail (par $\mathrm{UTA},+92 \%$ en Ha SAU et $+85 \%$ en UGB). Ainsi le poste amortissement rapporté à l'hectare passe de $136 €$ à $253 € /$ ha soit $+86 \%$ en 24 ans. De plus, cette hausse par ha est démultipliée par la croissance de la taille des exploitations, si bien que le montant des amortissements ramené par UTA gonfle de $5227 €$ à $18362 €$ soit $+250 \%$ (figure 8D). Dans une moindre mesure le montant des facteurs externes s'accroit de $25 \%$ par ha et de $133 \%$ par UTA. Les évolutions de l'augmentation de la productivité du travail $(+92 \%)$, des amortissements par UTA $(+250 \%)$ sans augmentation du revenu net sont à mettre en regard et seraient à analyser plus finement dans chaque contexte local pour en juger la pertinence (Veysset et al 2014).

\section{Conclusion}

Les systèmes bovins viande, importants dans quatre grands pays Européens, se sont développés depuis plus de 30 ans.
Dans un espace européen très varié avec des conditions pédoclimatiques extrêmement différentes, ces exploitations ont des structures de taille et de niveaux d'investissements également très divers. Chaque pays a fait des choix d'itinéraires contrastés en mettant l'accent soit sur la productivité du travail soit sur l'économie de charges. Mais les revenus nets moyens annuels restent tous dans une fourchette de 12 à $17 \mathrm{k} €$ par UTA avec des différences qui se réduisent entre pays. Ils restent quoi qu'il en soit parmi les plus faibles des professions agricoles sans que les crises marquantes puissent l'expliquer, et cela malgré des soutiens publics et de nombreuses adaptations structurelles et techniques.

Globalement, la filière bovins viande a perdu en compétitivité, comme l'ensemble des filières agricoles et agro-alimentaires en France (Butault et Requillart 2012 et 2015). Cela doit interroger pour l'avenir non seulement les professionnels, mais aussi les décideurs, voire les consommateurs et les citoyens. Ces productions sont indispensables à l'entretien et aux activités économiques et sociales des zones difficiles où peu d'alternatives existent, et leurs utilités agro-environnementales doivent être mieux reconnues. La prise en compte des coûts de production à l'échelle de l'élevage dans l'élaboration des prix de marché apparait donc indispensable pour l'avenir des élevages bovin viande. Quelques initiatives vont dans ce sens, mais quelles seraient les conséquences au niveau des consommateurs de prix qui rémunéreraient correctement les facteurs de production avec en premier lieu le travail et les risques pris (Veysset et al 2016) ? Et quelle répartition de valeurs ajoutées pour une vraie politique partenariale (cf. Cerles et al, ce numéro) ?

\section{Remerciements}

Les auteurs remercient particulièrement Alberto Bernués et Mireia Blanco-Alibes du C.I.T.A Aragon (Zaragoza) pour les informations concernant l'Espagne et Claire Mosnier pour celles sur l'Allemagne.

\section{Références}

Agabriel J., Brouard S., Devun J., Bastien D. Benoit M., D’hour P., Farrié J.P., Leclerc M.C., Pottier E., 2014. Guide de l'alimentation du troupeau bovin allaitant : Vaches, veaux et génisses de renouvellement. ISBN 978-2-36343-527-9. ISSN 2109-859X

Bébin D., Lherm M., Liénard G., 1995. Quels résultats techniques et économiques en grands troupeaux de vaches allaitantes? Le cas du Charolais, INRA Prod. Anim., 8, 213-226.

Butault J.P., Requillart V., 2012. L'agriculture et l'agro-alimentaire françaises à la recherche d'une compétitivité perdue. INRA Sciences Sociales, 4/5/2011, 1-4. http://prodinra.inra.fr/record/ $\underline{168855}$

Butault J.P., Requillart V., 2015. La compétitivité des filières alimentaires. In: Esnouf C., Fioramonti J., Laurious B. (Eds). L'alimentation à découvert, 110-111. À découvert. Paris, France : CNRS Editions.

Cerles A., Lherm M., Poux X., Agabriel J., 2017. Étude prospective des filières viande de ruminants du Massif central à 1'horizon 2050. In : Élevage bovin allaitant. Agabriel J., Renand G., Baumont R. (Eds). Dossier, INRA Prod. Anim., 30, 179-196.

Chatellier V., 2016. Le commerce extérieur de la France dans le secteur bovin. Renc. Rech. Rum., Paris, France, 23, 4p.

Chatellier V., Colson F., Fuentes M., Vard T., 2000. Les exploitations d'élevage herbivore dans l'Union Européenne. INRA Prod. Anim., 13, 201-213.

Dedieu B., Ingrand S., 2010. Incertitude et adaptation : cadres théoriques et application à l'analyse de la dynamique des systèmes d'élevage. In : Numéro spécial, Robustesse, rusticité, flexibilité, plasticité, résilience..., les nou- veaux critères de qualité des animaux et des systèmes d'élevage. Sauvant D., Perez J.M. (Eds). INRA Prod. Anim., 23, 81-90.

Dumont B., Dupraz P., Aubin J., Batka M., Beldame D., Boixadera J., Bousquet-Melou A., Benoit M., Bouamra-Mechemache Z., Chatellier V., Corson M., Delaby L., Delfosse C., Donnars C., Dourmad J.Y., Duru M., Édouard N., Fourat E., Frappier L., Friant-Perrot M., Gaigné C., Girard A., Guichet J.L., Haddad N., Havlik P., Hercule J., Hostiou N., HugueninElie O., Klumpp K., Langlais A., LemauvielLavenant S., Le Perchec S., Lepiller O., Letort E., Levert F., Martin B., Méda B., Mognard E.L., Mougin C., Ortiz C., Piet L., Pineau T., Ryschawy J., Sabatier R., Turolla S., Veissier I., Verrier E., Vollet D., van der Werf H., Wilfart A., 2016, Rôles, impacts et services issus des élevages en Europe. INRA, France, 1032 p.

Hocquette J.F., Chatellier V., 2011. Prospects for the European beef sector over the next 30 years. Anim. Frontiers, 1, 20-28.

Idele, 2015. Résultats des élevages bovins viande suivis par FCE Bovins croissance. Inosys ISBN 978-2-363-775-4.

Lemery B., Ingrand S., Dedieu B., Dégrange B., 2005. Agir en situation d'incertitude: le cas des éleveurs de bovins allaitants. Econ. Rurale, 288, 57-69.

Lherm M., Natier P., Veysset P., Boussemart J.P., 2015. Étude de l'évolution de la productivité avec la méthode des comptes de surplus dans des exploitations agricoles spécialisées en bovin viande : la productivité s'essouffle et ne permet pas de maintenir les revenus face aux prix malgré les soutiens. Congrès SFER Lille, décembre 2015 http://www.sfer.asso.fr/content/ download/6447/55280/version/1/file/jrss2015 lherm.pdf
Perrin J.B., Ducros C., Vinard J.L., Hendrikx P., Calavas D., 2011. Analyse de la mortalité bovine en France de 2003 à 2009, INRA Prod. Anim., 24, 235-244

Roguet C., Gaigné C., Chatellier V., Cariou S., Carlier M., Chenut R., Daniel K., Perrot C., 2015. Spécialisation territoriale et concentration des productions animales européennes. INRA Prod. Anim., 28, 5-22.

Sarzeaud P., Bécherel F., Perrot C., 2008. A classification of European beef farming systems. In EU beef farming systems and CAP regulations, EAAP Technic., 9, 23-32.

Stolz L., 2014. Cow-calf production in Germany Status quo and perspectives in the European context. Master thesis (English summary) Universitat Hohenheim Stuttgart, Braunschweig. $10 \mathrm{p}$.

Veysset P., Benoit M., Laignel G., Bébin D. Roulenc M., Lherm M., 2014. Analyse et déterminants de l'évolution des performances d'élevages bovins et ovins allaitants en zones défavorisés de 1990 à 2012. In : Spécificités de l'élevage de ruminants en montagne. Grosclaude J., Thibier M., Baumont R. (Eds). INRA Prod. Anim., 27, 49-64.

Veysset P., Lherm M., Roulenc M., Troquier C., Bébin D., 2015. Analyse diachronique de l'efficience technique des systèmes de production bovin viande. Baisse de la productivité des facteurs variables sur 23 ans. Econ. Rurale, 349-350, 149-169.

Veysset P., Lherm M., Natier P., Boussemart J.P., 2016. Formation et répartition des gains de productivité en élevage bovin viande. Qui sont les gagnants et les perdants sur les 35 dernières années ? Renc. Rech. Rum., 23, 11-14. 


\title{
Résumé
}

La production bovine allaitante européenne est surtout développée dans les zones herbagères plutôt défavorisées où peu d'alternatives existent. C'est le cas en France dans le Massif central, mais aussi en Espagne où elle s'est beaucoup accrue dans les montagnes sèches et au Royaume-Uni dans les collines d'Ecosse. Dans un contexte d'évolution à la hausse du coût des intrants et de stagnation des prix à la production, des stratégies bien différenciées depuis 2003/2005 ont été mises en évidence. En France et au Royaume-Uni, les éleveurs ont privilégié l'augmentation de la productivité de la main-d'œuvre. Cela s'est fait avec peu de conséquences sur les performances techniques, mais surtout en mobilisant davantage d'équipements et de mécanisation. Ces investissements, essentiellement en France, se sont souvent accompagnés d'endettement. A l'inverse en Espagne et en Irlande, les éleveurs ont moins modifié leurs dispositifs de production et ont eu une conduite économe en particulier sur le plan des investissements. Les politiques incitatives de soutien sont différentes : les primes à la vache allaitante demeurent en France et en Espagne alors que seules les primes à la surface existent au Royaume-Uni. Dans cet espace européen avec des conditions pédoclimatiques extrêmement différentes, des structures de taille et de niveaux d'investissements divers, quels que soient les stratégies choisies par les pays, les revenus nets moyens des éleveurs restent autour de $15 \mathrm{k} €$ par Unité de Travail Familial avec des différences qui se réduisent entre pays. Ces revenus restent inférieurs à ceux des autres productions agricoles.

\begin{abstract}
Status and trends of suckler beef production in France and in three European countries

European suckling cattle production is mainly developed in the rather disadvantaged grassland areas where few alternatives exist. This is the case in France in the Massif central, but also in Spain where it has increased greatly in the dry mountains and in the United Kingdom in the hills of Scotland. In the context of rising input costs and stagnating producer prices, well-differentiated strategies since 2003/2005 have been highlighted. In France and the United Kingdom, breeders have favored an increase in labor productivity. This has been done with little impact on technical performance but above all by mobilizing more equipment and mechanization. These investments, mainly in France, have often been accompanied by indebtedness. On the contrary, in Spain and Ireland, breeders have modified their production systems less and have been economically efficient, particularly in terms of investment. The support incentive policies are different: suckler cow premiums remain in France and Spain, whereas only the surface premiums exist in the United Kingdom. In the highly varied European area, with very different pedoclimatic conditions, different size structures and different levels of investment, irrespective of the major strategies chosen by the countries, the average net income of breeders remains around $15 \mathrm{k} €$ per FWU with a reduced differences between countries. These incomes remain lower than those of others agricultural productions.
\end{abstract}

LHERM M., AGABRIEL J., DEVUN J., 2017. État des lieux et évolutions de la production bovine allaitante en France et dans trois pays européens. In : Élevage bovin allaitant. Agabriel J., Renand G., Baumont R. (Eds). Dossier, INRA Prod. Anim., 30, 93-106. 